\title{
Therapeutic effect of MIPPSO in the thoracolumbar vertebra fracture
}

\author{
KEWEI REN ${ }^{1}$, JILEI TANG ${ }^{2}$, XUEFENG JIANG ${ }^{1}$, LUMING NONG $^{3}$ and YANQING GU ${ }^{4}$ \\ ${ }^{1}$ Department of Orthopedics, The Affiliated Jiangyin Hospital of Southeast University Medical School, Jiangyin, \\ Jiangsu 214400; ${ }^{2}$ Department of Orthopedics, Qidong People's Hospital, Nantong, Jiangsu 226200; \\ ${ }^{3}$ Department of Orthopedics, The Affiliated Changzhou No. 2 People's Hospital of Nanjing Medical University, \\ Changzhou, Jiangsu 213003; ${ }^{4}$ Department of Orthopedics, Nanjing First Hospital, \\ Nanjing Medical University, Nanjing, Jiangsu 210006, P.R. China
}

Received January 15, 2018; Accepted June 26, 2018

DOI: $10.3892 /$ etm.2018.6368

\begin{abstract}
The purpose of this study was to compare the efficacy of minimally invasive percutaneous pedicle screw osteosynthesis (MIPPSO) and traditional open pedicle screw osteosynthesis (TOPSO) in the treatment of thoracolumbar vertebra fracture. A retrospective case-control study was conducted in 120 patients with thoracolumbar vertebral fractures treated in the Affiliated Jiangyin Hospital of Southeast University Medical School (Jiangyin, China) from January 2013 to September 2014. They were randomly divided into two groups: MIPPSO and TOPSO groups with 60 cases in each group. The operation time, blood loss, incision length, post-operative bed rest time, hospital stay, visual analogue scale (VAS) pain scores and Oswestry disability index (ODI) scores before and after operation were recorded and analyzed. Inflammatory indexes including serum C-reactive protein (CRP) and creatine kinase (CK), the anterior vertebral height ratio and kyphosis Cobb's angle changes were also observed. The basic data of the two groups were similar, and there was no significant difference in the operation time between the two groups. The perioperative blood loss, length of incision, bed rest time and total hospital stay in the minimally invasive group were less than those in the open group. Levels of post-operative inflammation indicators such as CRP and CK were significantly higher than those of pre-operative $(\mathrm{P}<0.05)$, which was more obvious in the TOPSO group $(\mathrm{P}<0.05)$. VAS,
\end{abstract}

Correspondence to: Dr Luming Nong, Department of Orthopedics, The Affiliated Changzhou No. 2 People's Hospital of Nanjing Medical University, 29 Xinglongxiang Road, Tianning, Changzhou, Jiangsu 213003, P.R. China

E-mail: nongluming1977@hotmail.com

Dr Yanqing Gu, Department of Orthopedics, Nanjing First Hospital, Nanjing Medical University, 68 Changle Road, Nanjing, Jiangsu 210006, P.R. China

E-mail: cfzzu415@163.com

Key words: percutaneous pedicle screw, thoracolumbar fracture, minimally invasive
ODI scores, anterior vertebral height and Cobb's angle were significantly improved at three days, one and 12 months after surgery compared with those before operation. MIPPSO for the treatment of thoracolumbar fractures can achieve similar clinical effects with traditional incision surgery. In addition, it has the advantages of less trauma, less bleeding and shorter post-operative bed rest time and hospital stay.

\section{Introduction}

Thoracolumbar fracture is a common clinical traumatic vertebral trauma. Conservative treatment is mainly for simple vertebral compression fractures, while open reduction and internal fixation are often applied to patients with more than 1/2 high compression of the vertebral body or with reduced spinal canal sagittal diameter. However, there are some shortcomings in these two treatment types. Simple conservative treatment often causes complications such as instability of spine, chest and back pain, limited mobility, aggravation of compression and kyphosis, while the open surgery may lead to paravertebral muscle damage and denervation due to soft tissue exfoliation and distraction exposure (1). As a result, some patients have low confidence in the treatment or feel dissatisfied with the efficacy because of the intraoperative blood loss and post-operative residual back pain (2).

Therefore, how to get good curative effect with the lowest trauma becomes the focus of the research. It is in this background that minimally invasive surgery appears to overcome the shortcomings of traditional conservative treatment and open surgery, making it easier for patients to accept.

In this study, minimally invasive percutaneous pedicle screws osteosynthesis (MIPPSO) and traditional open pedicle screws osteosynthesis (TOPSO) were used to treat 120 patients with simple thoracolumbar fractures and achieved good results. MIPPSO has been proved not only safe, reliable and effective in curing thoracolumbar fracture, but also has the advantages of small trauma and rapid recovery after treatment.

\section{Patients and methods}

General information. A retrospective case-control study was conducted in 60 patients with single-stage thoracolumbar fracture 
in the Affiliated Jiangyin Hospital of Southeast University Medical School (Jiangyin, China) from January 2013 to September 2014 and treated with minimally invasive percutaneous pedicle screw fixation (the minimally invasive group). At the same time, 60 patients with thoracolumbar fracture undergoing conventional incision surgery were selected (the open group). As more patients underwent incision surgery during the same period than patients with minimally invasive surgery, we selected patients with similar condition and injury stage in these two groups. The two surgeries were performed by the same chief surgeon and his medical team, and all patients underwent follow-up of no less than one year with a mean follow-up of 14 months. In addition, the patients were all single thoracolumbar fracture with no symptoms of lower extremity nerve damage. This study was approved by the Ethics Committee of the Affiliated Jiangyin Hospital of Southeast University Medical School (Jiangyin, China) and the Affiliated Changzhou No. 2 People's Hospital with Nanjing Medical University (Changzhou, China). Signed informed consents were obtained from all participants before the study. The criteria of the enrolled patients were as follows: i) Patients with unstable thoracolumbar fracture; ii) patients with thoracolumbar fracture without injury of spinal or spinal nerve; iii) patients with non-burst fracture or thoracolumbar burst fracture without spinal canal involved; and iv) patients without spinal hematoma, foreign body in spinal canal.

Minimally invasive fixation group included 60 cases, 35 males and 25 females aged 23-56 with mean age of $42.8 \pm 10.8$ years. Injured segments were as follows: T11, 8 cases; T12, 10 cases; L1, 18 cases; L2, 8 cases; L3, 10 cases; and L4, 6 cases. Causes of injury were 22 cases falling from a height, 26 cases traffic accidents and 12 cases falling.

Open reduction and internal fixation group consisted of 60 cases, 36 males and 24 females aged 18-60, with mean age of 43.6 \pm 11.2 years. Injured segments were as follows: T11, 8 cases; T12, 10 cases; L1, 18 cases; L2, 8 cases; L3, 10 cases; and L4, 6 cases. Causes of injury were 24 cases falling from a height, 26 cases traffic accidents and 10 cases falling.

Surgical methods. Prone position was used in minimally invasive group after general anesthesia with in vitro positioning to determine and mark the injured vertebra and adjacent vertebra. The puncture needle was located at $1.0-2.0 \mathrm{~cm}$ next to the spinous process, then $1.5 \mathrm{~cm}$ skin incision was cut in each puncture position. As soon as the articular process was probed by hollow locator with a core needle, and location and angle confirmed by $\mathrm{C}$-arm fluoroscopy, we hammered the locator into the vertebral pedicle $\sim 1.0-1.5 \mathrm{~cm}$ deep. Then we pulled the needle out, inserted Kirschner wire of the same size as the core needle. Protection of the erector spina muscle was finished by the Kirschner wire, guiding three sleeve channels through it; the inner two layers of sleeves were then extracted. After the cutting cone piercing the vertebral pedicle, we pierced the open cone into thoracic vertebra for $3.5 \mathrm{~cm}$ and lumbar spine for $4.0 \mathrm{~cm}$, then screwed the upper and lower pedicle screw, respectively. According to the angle needed to be reset, we pre-bended the longitudinal connecting rod and penetrated into the longitudinal bar after percutaneous stripping of muscle under the $\mathrm{C}$-arm fluoroscopy. The plane mark showing concave toward was applied in the two ends of the connecting rod, which makes it easy to find if the bar was in the correct position and
Table I. Comparisons of general conditions of two groups of patients.

\begin{tabular}{lccc}
\hline Items & $\begin{array}{c}\text { MIPPSO } \\
\text { group }\end{array}$ & $\begin{array}{c}\text { TOPSO } \\
\text { group }\end{array}$ & P-value \\
\hline Sex (male/female, $\mathrm{n})$ & $35 / 25$ & $36 / 24$ & $>0.05$ \\
Age $($ mean \pm SD, years $)$ & $42.8 \pm 10.8$ & $43.6 \pm 11.2$ & $>0.05$ \\
BMI $\left(\mathrm{kg} / \mathrm{m}^{2}\right)$ & $23.3 \pm 0.72$ & $24.0 \pm 0.68$ & $>0.05$ \\
Injured segment $(\mathrm{n})$ & & & \\
T11 & 8 & 8 & \\
T12 & 10 & 10 & \\
L1 & 18 & 18 & 1 \\
L2 & 8 & 8 & \\
L3 & 10 & 1 & \\
L4 & 6 & 6 & \\
\hline
\end{tabular}

The general conditions, sex distribution, age, BMI and injured segment of two groups of patients were statistically analyzed, showing no significant differences $(\mathrm{P}>0.05)$. Baseline characteristics were comparable between two groups. MIPPSO, minimally invasive percutaneous pedicle screw osteosynthesis; TOPSO, traditional open pedicle screw osteosynthesis.

direction, then the vertebral body was propped up. Finally the nut was tightened and fixed with the perspective satisfactorily; screw tail was then removed and the wound sutured.

Patients in open reduction group took prone position after general anesthesia, and a posterior midline incision was made, centered on the injured level. Wiltse approach (3) was performed from the gap between the multi-muscle and the longest muscle to reveal the injured vertebra and vertebral facet joint. The location of pedicle screw implantation was determined by 'herringbone crest'. Posterior fixation with a screw rod was directly performed on subjects who suffered from thoracolumbar burst fractures without neurological symptoms or simple compression fractures. Once the height of the vertebral body was satisfactory after being propped up, the wound was flushed and the drainage tube placed, incision was sutured layer by layer.

Evaluation indicators. Perioperative data: Perioperative and follow-up indicators are as follows: the operation time, intraoperative and post-operative blood loss, the incision length, post-operative ambulation time and hospital stay, were all used to evaluate the conditions of the subjects.

Imaging evaluation. i) The anterior vertebral height ratio was measured pre-operatively at three days, one and 12 months post-operatively [anterior height ratio of vertebral body $=$ anterior height of fractured vertebral body/reference anterior height of vertebral body x $100 \%$; reference anterior height of vertebral body $=$ (anterior height of superior vertebra + anterior height of inferior vertebral body)/2]; ii) Cobb's angle was measured respectively, pre-operative, and 3 days, one and 12 months after the patients underwent the operation.

Inflammation-related indicators. Serum levels of C-reactive protein (CRP) and creatine kinase $(\mathrm{CK})$ were measured pre-operatively, 24 and $48 \mathrm{~h}$, post-operatively. 

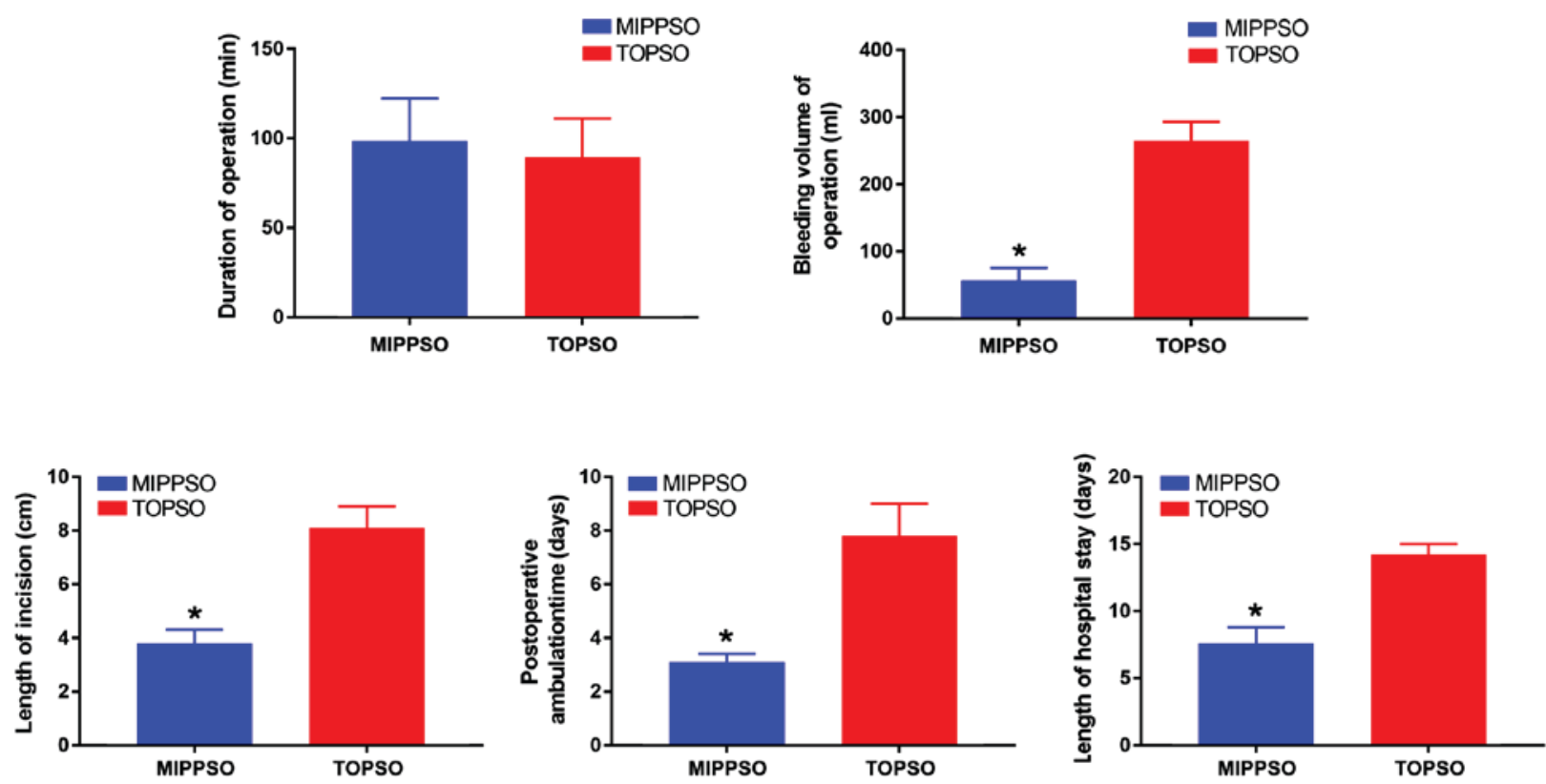

Figure 1. Comparison of perioperative parameters of the two groups of patients, ${ }^{*} \mathrm{P}<0.05$.
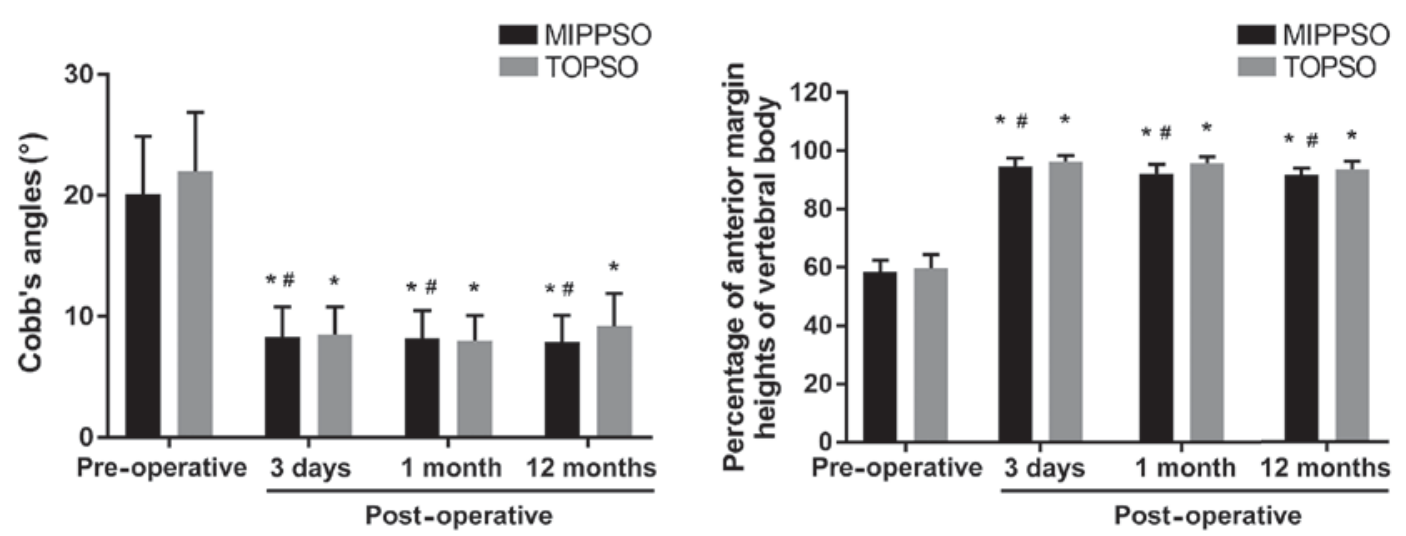

Figure 2. Comparisons of Cobb's angles and anterior margin heights of vertebral body (imaging index) of the two groups of patients. Compared to the pre-operation, ${ }^{*} \mathrm{P}<0.05$; compared to the TOPSO group, ${ }^{\prime} \mathrm{P}>0.05$. TOPSO, traditional open pedicle screw osteosynthesis.

Assessment of pain. The pain degree was evaluated by visual analog scale (VAS), and the patient's symptoms were assessed by Oswestry disability index (ODI).

Statistical analysis. The data were analyzed by SPSS 19.0 (SPSS, Inc., Chicago, IL, USA) statistical software. Enumeration data were analyzed with $\chi^{2}$ test and measurement data presented as mean \pm standard deviation (mean \pm SD) with t-test. $\mathrm{P}<0.05$ was considered to indicate a statistically significant difference.

\section{Results}

Comparison of basic data between the two groups. Sample number, age, sex, body mass index (BMI) and injured segments of patients in the two groups have no significant difference (Table I).

Perioperative data. The operation time in the minimally invasive group was slightly longer than that in the traditional incision group, but the difference was not statistically significant. As for the perioperative blood loss (including intraoperative blood loss and post-operative drainage), surgical incision length, post-operative bed rest time and total hospital stay, the minimally invasive group was superior to the open incision group. The difference was statistically significant (Fig. 1).

Imageological assessment. The height of the anterior vertebral body and Cobb's angle of kyphosis were significantly improved in three days, one and 12 months after operation in both groups. Paired t-test was used to analyze the post-operative and pre-operative time-points in both groups; as a result, the difference was statistically significant $(\mathrm{P}<0.05)$. Two independent samples t-test was used to analyze the difference between the two groups at the same time-points, and the difference was not statistically significant $(\mathrm{P}>0.05)$ (Fig. 2).

Inflammation-related indicators. There was no conspicuous difference in serum CRP and CK between the two groups before operation $(\mathrm{P}>0.05)$. However, at 24 and $48 \mathrm{~h}$ after 

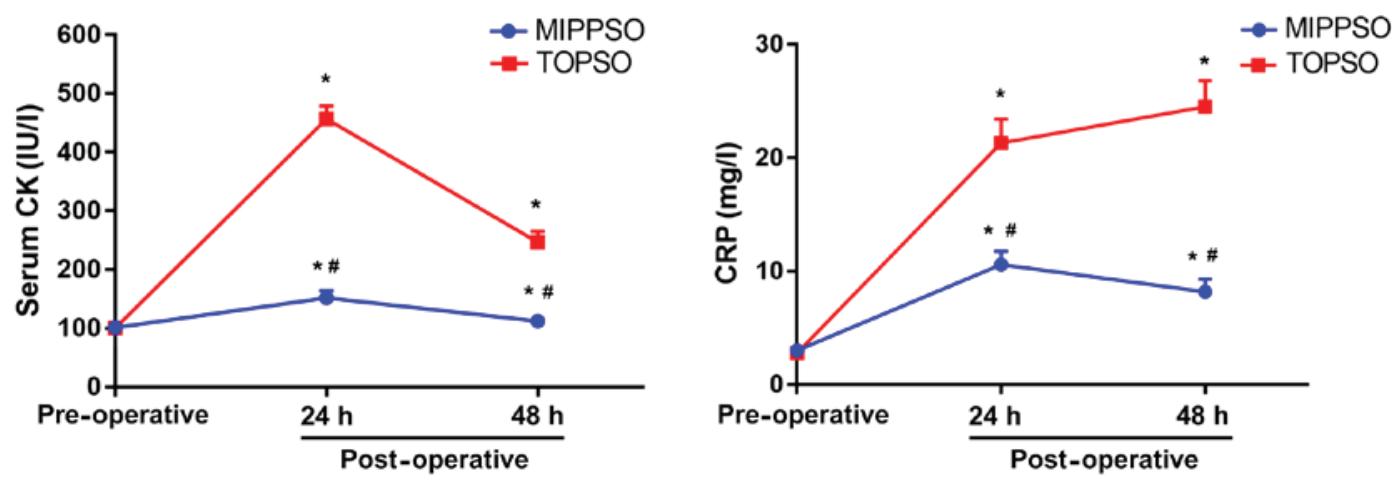

Figure 3. Comparison of pre-operative and post-operative serum CRP and serum CK levels of two groups of patients. Compared to the pre-operation, ${ }^{*} \mathrm{P}<0.05$; compared to the TOPSO group, ${ }^{\sharp} \mathrm{P}<0.05$. CRP, C-reactive protein; CK, creatine kinase; TOPSO, traditional open pedicle screw osteosynthesis.
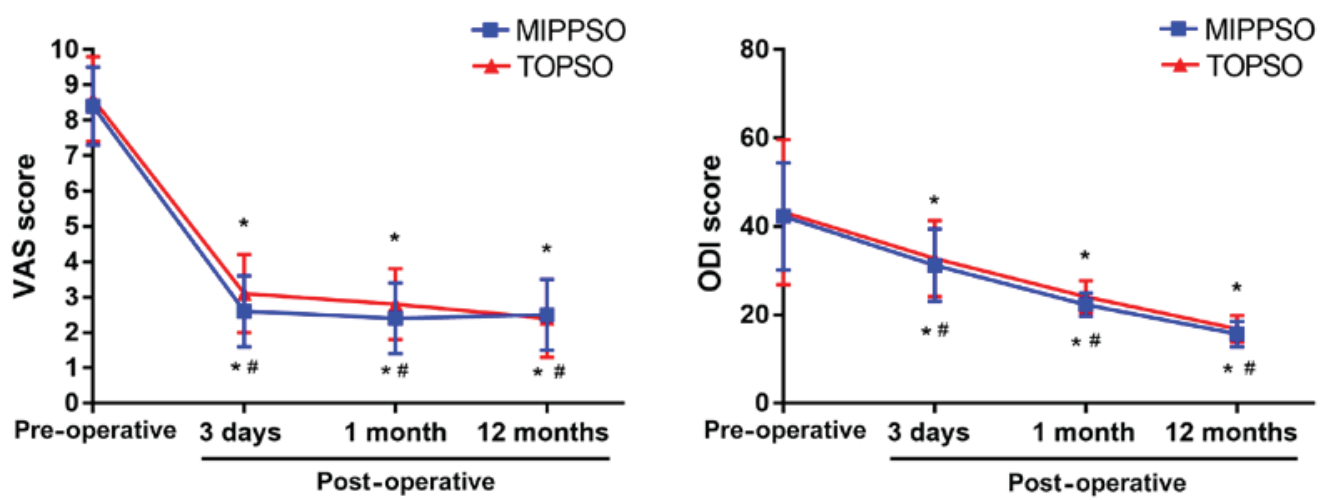

Figure 4. Comparison of pre-operative and post-operative VAS pain scores and ODI scores of two groups of patients. Compared to the pre-operation, ${ }^{*} \mathrm{P}<0.05$; compared to the TOPSO group, ${ }^{\sharp} \mathrm{P}>0.05$. VAS, visual analogue scale; ODI, Oswestry disability index; TOPSO, traditional open pedicle screw osteosynthesis.

operation, serum CRP and CK of patients in the minimally invasive group were significantly lower than those in open incision group $(\mathrm{P}<0.05)$ (Fig. 3).

Pain assessment. There were no significant differences in VAS and ODI scores between the two groups before surgery $(\mathrm{P}>0.05)$. However, VAS and ODI scores of 3 days, 1 month and 12 months after operation were significantly improved compared with those before operation. Paired t-test was used to analyze the post-operative and pre-operative time-points in both groups, and the difference was statistical significant $(\mathrm{P}<0.05)$. Two independent samples t-test was used to analyze the difference between the two groups at the same time-points, and the difference was not statistically significant ( $\mathrm{P}>0.05$ ) (Fig. 4).

\section{Discussion}

In the past, TOPSO were mainly applied for the treatment of lumbar and thoracic fractures, but clinical study found significant lumbar dysfunction and pain at follow-up after surgery. Most scholars believe that muscle fibrosis caused by long time traction of muscle fiber can give rise to the patient's muscle function decline, muscle fiber edema, and nerve changes (4).

With the continuous development of minimally invasive medical technology, nerve damage can be avoided in patients treated with MIPPSO, so that MIPPSO is increasingly widely used in treatment of lumbar and thoracic fractures. The pathological area in which the nail is put in does not contain the main dominant nerve. In addition, this way of treatment does not require a long time separation and traction of muscle fibers, avoiding damage to muscle function and muscle fibers as well as promoting post-operative recovery of patients (5). Lowery and Kulkarni (6) conducted percutaneous minimally invasive internal fixation and traditional open internal fixation for the treatment of thoracolumbar fractures. Comparative study showed that the former operation is simple, safe and reliable with less trauma, less bleeding, faster recovery, shorter hospital stay, less complications after internal fixation, under the premise of strictly taking charge of indications.

Moreover, the effect of deformity correction and internal fixation in the injured vertebral of MIPPSO was equivalent to that of traditional open reduction.

Magerl (7) used MIPPSO for the treatment of thoracolumbar fractures; as a result, all patients had a good reduction and the vertebral height maintained well at the final follow-up. Oh et al (8) demonstrated that accuracy of screw placement of percutaneous minimally invasive fixation system had no difference with open reduction and internal fixation, which further proved the safety of the former. Prokop et al (9) compared the efficacy of percutaneous minimally invasive internal fixation system and traditional open reduction and internal fixation in the treatment of unstable spinal fractures. The results showed that operative time and hospital stay were significantly shorter in the minimally invasive group. Schmidt et al (10) proved that transdermal fixation system has advantages for the treatment 
of patients with acute thoracolumbar spine trauma. Its short operative time and minimal blood loss make it suitable for the treatment of patients who cannot tolerate traditional surgery due to severe trauma or high surgical risk. The results of our study also indicate that percutaneous minimally invasive internal fixation system has the merits of short treatment time, less intraoperative blood loss, shorter hospital stay, and equal orthodontic effect to open reduction and internal fixation. This proves again that the minimally invasive system is feasible and practical.

Compared with open reduction and internal fixation, requirements for surgeon's skill are higher in the process of percutaneous minimally invasive internal fixation. The operating points are as follows: i) The most important is positioning before internal fixation, so the $\mathrm{C}$-arm performance should be checked and adjusted to the clearest state. ii) C-arm should be adjusted to horizontal position after patients adopt prone position. With $\mathrm{C}$-arm fluoroscopy, the positive phase requires the spinous process to be located in the bilateral pedicle center to avoid puncture errors. iii) As soon as the U-shaped groove gets to the articular process, the screw should be stopped to maintain the universal property. Putting the screw in too deeply will lead to the difficulty of linking the connecting rod. iv) Screw extension rod should be kept universal and the tail end kept a similar length as far as possible for complete match. v) Intraoperative distraction reduction is limited, so that it needs to be repeated a few times. Intraoperative pressure reduction or traction reduction are applied with distraction. This group of patients are all cooperated with manual distraction.

In addition to the many advantages, percutaneous minimally invasive internal fixation systems also have shortcomings: i) Although percutaneous minimally invasive internal fixation systems provide ancillary tools for longitudinal distraction and compression, the effect of distraction reduction of it is not as good as open reduction and internal fixation due to its short operable distance (11). Therefore, it must be cooperated with manual pressing reduction clinically. ii) Its screws are universal nail and tie rod that cannot be assembled between the connecting rod, zygapophysis fusion cannot be operated in surgery, which makes the loss of the mid-long term correction of vertebral height higher than the open reduction group (12). In addition, biomechanical measurements show that the strength of post-operative vertebral in the direction of flexion and extension is weak. iii) The system is currently mainly used for simple vertebral compression fracture and slipping cases without decompression, so its indications are relatively narrow. However, it is encouraging that with the improvement of minimally invasive operation and the joint application with minimally invasive devices, some scholars have collaborated with the Quadrant system, MED system to successfully accomplish spinal canal decompression and fusion, and good effects have been achieved $(13,14)$.

In conclusion, compared with the traditional incision pedicle screw fixation in the treatment of lumbar and thoracolumbar fractures, the main advantages of minimally invasive percutaneous pedicle screw internal fixation are as follows: shorter operation time, reduced surgical incision and amount of bleeding, shorter hospital stay and reduced patient trauma. Furthermore, it can also promote patients' recovery as well as reduce their post-operative pain. According to patients who underwent MIPPSO, their lumbar function recovery is better than that of those who underwent TOPSO and they exhibit evident lumbar physiological structure recovery after surgery, which suggests that the use of MIPPSO worth further promotion in clinical application.

In conclusion, MIPPSO for the treatment of thoracolumbar fractures can achieve similar clinical effects as traditional incision surgery. In addition, it has the advantages of less trauma, less bleeding and shorter post-operative bed rest time and hospital stay.

\section{Acknowledgements}

Not applicable.

\section{Funding}

This study was supported by the National Natural and Science Foundation (81501874), Jiangsu Province Health and Family Planning Commission Foundation (Q201511 and QNRC2016139) (all foundations to KR); The Project of Invigorating Health Care through Science, Technology and Education (Jiangsu Provincial Medical Youth Talent) and Changzhou High-level Medical Talents Training Project (2016CZBJ029) (all foundations to LN).

\section{Availability of data and materials}

All data generated or analyzed during this study are included in this published article.

\section{Authors' contributions}

KR and JT performed the operations for the patients. XJ and LN collected the patient data. KR and YG analyzed the patient data. All authors have read and approved the final manuscript.

\section{Ethics approval and consent to participate}

This study was approved by the Ethics Committee of the Affiliated Jiangyin Hospital of Southeast University Medical School (Jiangyin, China) and the Affiliated Changzhou No. 2 People's Hospital with Nanjing Medical University (Changzhou, China). Signed informed consents were obtained from the patients or guardians.

\section{Patient consent for publication}

Not applicable.

\section{Competing interests}

The authors declare that they have no competing interests.

\section{References}

1. Boelderl A, Daniaux H, Kathrein A and Maurer H: Danger of damaging the medial branches of the posterior rami of spinal nerves during a dorsomedian approach to the spine. Clin Anat 15: 77-81, 2002.

2. Agrawal A, Mizuno J, Kato Y, Inoue T and Sano H: Minimally invasive pedicle screw placement in a case of L4 fracture: Case report with review of literature. Asian J Neurosurg 5: 64-69, 2010

3. Wiltse LL and Spencer CW: New uses and refinements of the paraspinal approach to the lumbar spine. Spine 13: 696-706, 1988. 
4. Rodgers WB, Gerber EJ and Patterson J: Intraoperative and early postoperative complications in extreme lateral interbody fusion: An analysis of 600 cases. Spine 36: 26-32, 2011.

5. Wang MY, Vasudevan R and Mindea SA: Minimally invasive lateral interbody fusion for the treatment of rostral adjacent-segment lumbar degenerative stenosis without supplemental pedicle screw fixation. J Neurosurg Spine 21: 861-866, 2014.

6. Lowery GL and Kulkarni SS: Posterior percutaneous spine instrumentation. Eur Spine J 9 (Suppl 1): S126-S130, 2000.

7. Magerl FP: Stabilization of the lower thoracic and lumbar spine with external skeletal fixation. Clin Orthop Relat Res 189: 125-141, 1984

8. Oh HS, Kim JS, Lee SH, Liu WC and Hong SW: Comparison between the accuracy of percutaneous and open pedicle screw fixations in lumbosacral fusion. Spine J 13: 1751-1757, 2013.

9. Prokop A, Lohlein F, Chmielnicki M and Volbracht J: Minimally invasive percutaneous instrumentation for spine fractures Unfallchirurg 112: 621-628, 2009 (In German).

10. Schmidt OI, Strasser S, Kaufmann V, Strasser E and Gahr RH: Role of early minimal-invasive spine fixation in acute thoracic and lumbar spine trauma. Indian J Orthop 41: 374-380, 2007.
11. Wang HW, Li CQ, Zhou Y, Zhang ZF, Wang J and Chu TW: Percutaneous pedicle screw fixation through the pedicle of fractured vertebra in the treatment of type A thoracolumbar fractures using Sextant system: An analysis of 38 cases. Chin J Traumatol 13: 137-145, 2010.

12. Krüger A, Rammler K, Ziring E, Zettl R, Ruchholtz S and Frangen TM: Percutaneous minimally invasive instrumentation for traumatic thoracic and lumbar fractures: A prospective analysis. Acta Orthop Belg 78: 376-381, 2012.

13. Kang H, Cai X, Xu F and Huang Y: Effectiveness of combined treatment of lumbar spondylolisthesis with MED, Quadrant, and Sextant-R systems. Zhongguo Xiu Fu Chong Jian Wai Ke Za Zhi 27: 399-403, 2013 (In Chinese)

14. Sairyo K, Sakai T and Yasui N: Minimally invasive technique for direct repair of pars interarticularis defects in adults using a percutaneous pedicle screw and hook-rod system. J Neurosurg Spine 10: 492-495, 2009.

c) (i) (9) This work is licensed under a Creative Commons

EY NO NO Attribution-NonCommercial-NoDerivatives 4.0 International (CC BY-NC-ND 4.0) License. 